7. Hara M, Ma T, Verkman AS. Selectively reduced glycerol in skin of aquaporin-3-deficient mice may account for impaired skin hydration, elasticity, and barrier recovery. J Biol Chem 2002; 277: 46616-21.

8. Zheng X, Bollinger Bollag W. Aquaporin 3 colocates with phospholipase $\mathrm{d} 2$ in caveolin-rich membrane microdomains and is downregulated upon keratinocyte differentiation. J Invest Dermatol 2003; 121: 1487-95.
9. van Deurs B, Roepstorff K, Hommelgaard AM, et al. Caveolae: anchored, multifunctional platforms in the lipid ocean. Trends Cell Biol 2003; 13: 92-100.

10. Murray NR, Fields AP. Phosphatidylglycerol is a physiologic activator of nuclear protein kinase C. J Biol Chem 1998; 273: 11514-20.

11. Denning MF. Epidermal keratinocytes: regulation of multiple cell phenotypes by multiple protein kinase C isoforms. Int J Biochem Cell Biol 2004; 36: 1141-6.
12. Nielsen S, King LS, Christensen BM, et al. Aquaporins in complex tissues. II. Subcellular distribution in respiratory and glandular tissues of rat. Am J Physiol 1997; 273: 1549-61.

13. Parsa R, Yang A, McKeon F, et al. Association of $p 63$ with proliferative potential in normal and neoplastic human keratinocytes. J Invest Dermatol 1999; 113: 1099-105.

\title{
NOUVELLE
}

\section{Dépistage IRM du cancer du sein chez les femmes à haut risque: une avancée dans le dépistage?}

Martine Meunier, Dominique Stoppa-Lyonnet

$>$ Cinq pour cent des cas de cancer du sein sont liés à une prédisposition génétique majeure. Les mutations constitutionnelles des gènes BRCA1 et BRCA2 sont à l'origine de $65 \%$ des cas de prédisposition [1]. On estime qu'une femme sur 400 dans la population est porteuse d'une mutation de l'un ou de l'autre de ces gènes [2]. Leur risque de cancer du sein cumulé à l'âge de 70 ans est de l'ordre de $65 \%$ et $45 \%$ en cas de mutation de respectivement, BRCA1 et BRCA2. À 50 ans, il est déjà de $38 \%$ et $16 \%$ [3], soit entre 8 et 20 fois supérieur à celui des femmes de la population générale auxquelles on propose de participer au dépistage organisé. Du fait de l'augmentation significative du risque dès 30 ans, la prise en charge des femmes à haut risque doit débuter à cet âge, voire dès 25 ans lorsqu'un premier cas est survenu dans une famille avant l'âge de 30 ans. Aujourd'hui, en l'absence de chimioprévention et à l'alternative d'une chirurgie prophylactique mutilante et irréversible, seule la surveillance mammaire peut se discuter. La densité mammaire visible sur les clichés mammographiques est fonction de l'âge: les seins des femmes jeunes sont plus denses, ce qui a pour conséquence une sensibilité de dépistage moindre de la mammographie. En 1998 et lors de sa mise à jour en 2004, un rapport d'expert a recommandé de réaliser un examen clinique tous les six mois, une mammographie annuelle dès l'âge 30 ans, généralement couplée à une échographie [4, 5]. Néanmoins, plusieurs études ont montré que, malgré cette surveillance, il y avait jusqu'à $50 \%$ de cancers d'intervalle, c'est-àdire des cancers détectés cliniquement par une masse palpable entre deux mammographies [6-8] (on attendrait que le diagnostic soit fait par la mammographie au stade infraclinique). Ces mauvaises performances diagnostiques ont conduit certains à compléter le bilan annuel par une imagerie par résonance magnétique (IRM). Depuis une dizaine d'années, I'IRM s'est imposée dans le diagnostic des cancers du sein infiltrants. Elle peut être complétée par une injection intraveineuse de produit de contraste qui permet l'étude de la vascularisation du tissu mammaire et de la néovascularisation associée aux cancers. Si cet examen complémentaire est utilisé en pratique courante pour documenter une anomalie radiologique d'aspect complexe, son interprétation peut être difficile et son utilisation dans le cadre du dépistage n'a jamais été validée. En effet, devant une prise de contraste, parfois de très petite taille, il est nécessaire de distinguer une lésion maligne d'une lésion bénigne (fibroadénome, mastose fibrokystique) et d'une image qui correspond à une structure anatomique (petit ganglion intramammaire, vaisseau). Par ailleurs, les critères d'interprétation n'ont pas encore été validés sur de grandes séries. En conséquence, la spécificité de l'IRM, c'est-à-dire sa capacité de classer comme bénignes des lésions effectivement bénignes, est faible. Deux études récentes ont examiné l'apport de l'IRM chez les femmes à haut risque de cancer du sein, défini par la présence d'une mutation de BRCAl/2 $[9,10]$. La sensibilité de I'IRM apparaît très supérieure à celle de la mammographie et de l'échographie (Tableau I): la taille moyenne des tumeurs détectées par IRM est plus petite. Ces résultats doivent être considérés cependant avec prudence. $\varepsilon$ n effet, dans l'étude de $\varepsilon$. Warner, avec une sensibilité de I'IRM à $77 \%, 5$ cancers sur 22 n'ont pas été identifiés: un cas de cancer d'intervalle à 7 mois du bilan d'imagerie, deux cancers visibles par la mammographie seule et deux autres par l'échographie seule. 
Ce résultat montre la complémentarité nécessaire de ces différents examens. Par ailleurs, la spécificité et la valeur prédictive positive de I'IRM sont beaucoup moins bonnes que celles de la mammographie, conduisant notamment à un nombre élevé de biopsies pour des lésions bénignes ( $14 \%$ dans l'étude de $\varepsilon$. Warner et al.). Si la relecture de la mammographie et de l'échographie après la détection d'une prise de contraste à l'IRM ne permet pas d'orienter les biopsies nécessaires au diagnostic de certitude de lésion maligne, ces biopsies doivent être réalisées avec un repérage sous IRM. Ce repérage est complexe et nécessite une antenne particulière, en général disponible seulement dans les centres spécialisés. Il a été montré que $75 \%$ des prises de contraste vues en IRM n'étaient pas visibles en échographie et, parmi celles-ci, $15 \%$ correspondaient à un cancer [11]. Ainsi, on peut retenir que la prise en charge des résultats de l'ensemble de ces examens nécessite des équipes spécialisées en sénologie interventionnelle.

\begin{tabular}{|c|c|c|c|c|c|}
\hline & $\begin{array}{l}\text { Nombre de cas } \\
\text { (BRCA1/2) }\end{array}$ & $\begin{array}{c}\text { Nombre de cancers } \\
\text { identifiés }^{1}\end{array}$ & $\begin{array}{c}\text { Sensibilité } \\
(\%)\end{array}$ & $\begin{array}{l}\text { Spécificité }^{3} \\
(\%)\end{array}$ & $\begin{array}{l}\text { Valeur prédictive } \\
\text { positive }^{4}(\%)\end{array}$ \\
\hline [9] & 236 & 22 & & & \\
\hline IRM & & & 77,0 & 95,0 & 46,0 \\
\hline Mammographie & & & 36,0 & 99,8 & 88,9 \\
\hline Échographie & & & 33,0 & 96,0 & 29,0 \\
\hline [10] & 1909 dont & 51 & & & \\
\hline IRM & 358 & & 71,1 & 90,0 & 32,3 \\
\hline Mammographie & ie $B R C A 1 / 2$ & & 40,0 & 95,0 & 47,8 \\
\hline
\end{tabular}

Tableau I. Résultats des techniques de dépistage du cancer du sein chez des patientes à haut risque, obtenus à partir de deux études $[9,10]$. ${ }^{1}$ Le nombre de cancers identifiés dans les deux études n'est pas similaire du fait de la définition des risques fondée en partie sur la seule histoire familiale dans l'étude de M. Kriege et al. [10] et du fait du recul du suivi différent entre les deux études. ${ }^{2}$ Sensibilité: (nombre de vrais positifs/nombre de vrais positifs + nombre de faux négatifs) x 100. ${ }^{3}$ Spécificité: (nombre de vrais négatifs/nombre de vrais négatifs + nombre de faux positifs) $\times 100 .{ }^{4}$ Valeur prédictive positive: (nombre de vrais positifs/nombre de vrais positifs + nombre de faux positifs) $\times 100$.

\section{RéFÉRENCES}

1. Ford D, Easton DF, Stratton M, et al. Genetic heterogeneity and penetrance analysis of the BRCAl and BRCA2 genes in breast cancer families. The Breast Cancer Linkage Consortium. Am J Hum Genet 1998; 62: 676-89.

2. Antoniou AC, Pharoah PD, McMullan G, et al. A comprehensive model for familial breast cancer incorporating $B R C A 1, B R C A 2$ and other genes. $\mathrm{Br}$ J Cancer 2002; 86: 76-83.

3. Antoniou A, Pharoah PD, Narod S, et al. Average risks of breast and ovarian cancer associated with BRCAI or BRCA2 mutations detected in case series unselected for family history: a combined analysis of 22 studies. Am J Hum Genet 2003; 72 : $1117-30$.
4. Eisinger F, Alby N, Bremond A, et al. Recommendations for the management of women with a genetic risk for developing cancer of the breast and/or the ovary. Bull Cancer 1999; 86: 307-13.

5. Eisinger F, Bressac B, Castaigne D, et al. Identification et prise en charge des prédispositions héréditaires aux cancers du sein et de l'ovaire. Bull Cancer 2004; 91: 656 .

6. Brekelmans CT, Seynaeve C, Bartels CC, et al. Effectiveness of breast cancers surveillance in $B R C A l / 2$ gene mutation carriers and women with high familial risk. J Clin Oncol 2001; 19: 924-30.

7. Scheuer L, Kauff N, Robson M, et al. Outcome of preventive surgery and screening for breast and ovarian cancer in BRCA mutation carriers. J Clin Oncol 2002; 20 : 1260-8.
En conclusion, le bénéfice de la surveillance par IRM ne pourra être retenu que lorsqu'il aura été montré qu'elle est associée à une diminution de la mortalité. De plus, des questions restent à résoudre comme la combinaison et la périodicité des examens (mammographie et IRM annuelles, réalisées avec un décalage de six mois l'une par rapport à l'autre), l'assiduité des femmes à cette surveillance qui risque, à terme, d'être obérée par des examens invasifs itératifs, et, enfin, d'un point de vue socioéconomique, son coût ainsi que celui des examens induits. Les résultats des études de $\varepsilon$. Warner et $M$. Kriege nous encouragent à proposer, dans un cadre d'évaluation, un suivi IRM chez les patientes porteuses d'une altération d'un gène $B R C A[9,10]$. II faut cependant rester prudent chez les femmes dont le risque est défini par la seule histoire familiale et chez lesquelles l'incidence attendue des cancers est quatre fois moins importante que chez les femmes porteuses d'une mutation BRCA. $\varepsilon$ n effet, dans ces conditions, la mauvaise valeur prédictive positive de I'IRM est, au moins aujourd'hui, une limite à son utilisation en dépistage. $\diamond$ MRI for breast-cancer screening in women with predisposition: an advance in detection?
8. Komenaka IK, Ditkoff BA, Joseph KA, et al. The development of interval breast malignancies in patients with BRCA mutations. Cancer 2004; 100: 2079-83.

9. Warner $\varepsilon$, Plewes $D B$, Hill KA, et al. Surveillance of $B R C A 1$ and $B R C A 2$ mutation carriers with magnetic resonance imaging, ultrasound, mammography, and clinical breast examination. JAMA 2004; 292 : 1317-25.

10. Kriege M, Brekelmans CT, Boetes C, et al. Efficacy of $M R I$ and mammography for breast-cancer screening in women with a familial or genetic predisposition. $N$ Engl J Med 2004; 351: 427-37.

11. LaTrenta LR, Menell JH, Morris EA, et al. Breast lesions detected with MR imaging: utility and histopathologic importance of identification with US. Radiology 2003; 227 : 856-61 